\title{
Self-consistent theory of turbulent transport in the solar tachocline
}

\section{Anisotropic turbulence ${ }^{\star}$}

\author{
Eun-jin Kim
}

\author{
Department of Applied Mathematics, University of Sheffield, Sheffield, S3 7RH, UK \\ e-mail: e.kim@sheffield.ac.uk
}

Received 1 April 2005 / Accepted 24 June 2005

\begin{abstract}
We present a self-consistent theory of turbulent transport in the solar tachocline by taking into account the effect of the radial differential rotation on turbulent transport. We show that the shearing by the radial differential rotation leads to reduction in turbulent transport of particles and momentum and the amplitude of turbulent flow via shear stabilization. The degree of reduction depends on the direction as well as the quantity that is transported. Specifically, particle transport in the vertical (radial) direction, orthogonal to the shear flow, is reduced with the scaling $\propto \mathcal{A}^{-2}$ while it is less reduced in the horizonal plane with the scaling $\propto \mathcal{A}^{-4 / 3}$. Here, $\mathcal{A}$ is shearing rate, representing the radial differential rotation. A similar, but weaker, anisotropy also develops in the amplitude of turbulent flow. The results suggest that the radial differential rotation in the tachocline can cause anisotropy in turbulence intensity and particle transport with weaker turbulence in the radial direction even in the absence of density stratification and even when the turbulence is mainly driven radially by plumes from the convection zone. We also assess the efficiency of the transport by a meridional circulation by taking into account the interaction with the radial differential rotation. Implications for mixing and angular momentum transport in the solar interior is discussed.
\end{abstract}

Key words. turbulence - Sun: interior - Sun: rotation - Sun: abundances

\section{Introduction}

One of the outstanding problems in solar physics is to understand the processes by which the transport of the angular momentum and mixing of chemical species take place. To be consistent with observations, these processes should occur on time scales much shorter than those determined by molecular values (i.e., viscous or diffusive time scales) and also in regions where no fast transport is expected according to the standard solar model (e.g. Stix 1989). According to the latter, fast, turbulent transport is possible only in the convection zone ( $R \gtrsim 0.713 R_{\odot}$ ), where a vigorous convection takes place due to a unstable background stratification. Below the convection zone, a stable stratification is considered to lead to a quiet radiative interior without turbulent motion. Between these two regions lies a thin boundary layer - the so-called tachocline of thickness $\lessgtr 0.05 R_{\odot}$, through which the latitudinal differential rotation in the convection zone smoothly matches onto the uniform rotation in the radiative interior (e.g., Charbonneau et al. 1999). As a shear boundary layer, the tachocline contains both latitudinal and (strong) radial differential rotation. The density

* Appendices A and B are only available in electronic form at http://www. edpsciences.org stratification in this region is stable in the lower portion while possibly almost adiabatic in the upper part due to plumes penetrating from the convection zone (e.g. Gilman 2000; Rogers \& Glazmaier 2005)

In the case of the angular momentum transport, one of the greatest challenges is to explain how the radiative interior maintains the uniform rotation despite constant losses of the angular momentum via solar wind, etc from the surface over the course of solar evolution. This would require an efficient transport outside the convection zone on a time scale much shorter than solar age. On the other hand, the depletion of light element lithium on the solar surface, which is about a factor of 100 smaller than on Earth (Greenstein \& Richardson 1951), demands the existence of some kind of mixing outside the convection zone, which carries lithium down to $0.65 R_{\odot}$, little below the bottom of the tachocline, where lithium can be destroyed (e.g. Barnes et al. 1999; Pinsonneault 1997). Note that these light elements burn at high temperature, surviving only in the vicinity of the convection zone where the temperature is below the critical value for its destruction.

To understand these problems, it is necessary to identify physical processes, which lead to transport outside the convection zone, especially in the tachocline near the convection zone. 
In particular, how the transport in the convection zone is linked to that in the interior through the tachocline is crucial to understanding not only the present Sun but also the entire solar evolution. This is true even for the case of solar dynamos since the tachocline is considered to be the very site where toroidal fields are generated from poloidal magnetic fields via shearing ( $\Omega$ effect) (Moffatt 1978). Therefore, the dynamics of the tachocline, such as the presence and/or the origin of possible turbulence seems to play an unique role in the overall solar evolution.

A popular view is that turbulence in the tachocline, if any, should be strongly anisotropic with much less transport in the radial direction than in the horizontal plane due to the stable density stratification. While this anisotropic turbulence, interestingly, was invoked as a very mechanism for maintaining the identity of the tachocline itself against radiative broadening over the age of the Sun (Spiegel \& Zahn 1992), various (hydrodynamic and magnetohydrodynamic) instabilities of the latitudinal differential rotation (e.g. Gilman \& Fox 1997; Dikpati \& Gilman 1999; Gilman \& Dikpati 2000; Cally et al. 2003) have been proposed as a source of anisotropic turbulence.

In the absence of turbulence, transport can still be mediated by meridional circulation. In particular, the latter may carry light elements from the convection zone down to radiative interior on time scale which is sufficiently short. While a recent work by Gilman \& Miesch (2004) suggested that the meridional circulation, which is observed near the solar surface (Haber et al. 2000), is unlikely to persist deep below the convection zone in the present Sun, it is important to understand, in general, how the transport by meridional circulation is affected by turbulence and vice versus. The particle transport by meridional circulation can be particularly important for the depletion of light elements in massive stars such as lithium dip in F stars (e.g., Zahn 1992; Schatzman 1993; Pinsonneault 1997) or in the early evolution of the Sun (Pinsonneault et al. 1989) where the transport by a meridional circulation - the so-called Eddington-Sweet circulation (Eddington 1925; Vogt 1925; Sweet 1950) - is likely to be efficient in the interiors due to fast rotation. Interestingly, to obtain agreement with observed surface depletion of lithium, and at the same time to explain the angular momentum transport which seems to take place on a shorter time scale as compared to the mixing of lithium (e.g., Pinsonneault et al. 1989), anisotropic turbulence with a stronger horizontal turbulence was invoked to reduce the mixing by a meridional flow (Chaboyer \& Zahn 1992; Zahn 1992).

There is one key missing physics in studying these problems, which has been overlooked by virtually all the previous authors and in the traditional solar modelling. This is the remarkable effect of a stable shear flow on turbulent transport, namely turbulence regulation (or shear stabilization) (e.g. Burrell 1997; Hahm 2002; Kim 2004). When a shear flow acts on a (turbulent) eddy, it advects its different parts at different rates, distorting its shape and generating small scales in the direction orthogonal to the flow. This process continues until the eddy becomes disrupted by dissipation (see Fig. 1). As a result of this shearing process, the transport orthogonal to the shear flow, as well as the intensity of turbulence, is reduced
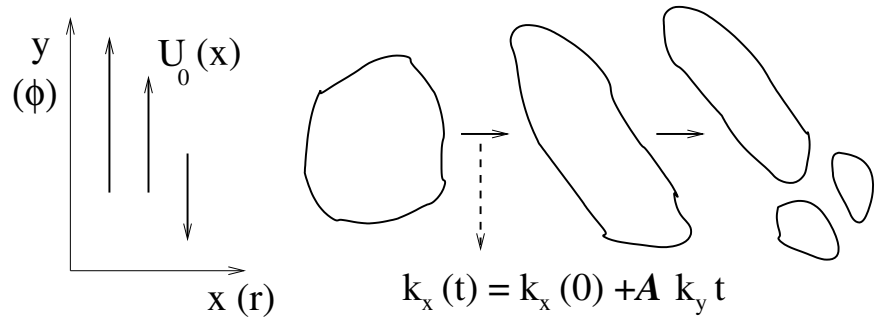

Fig. 1. Tilting and break-up of (isotropic) turbulent eddy due to the shearing by shear flow $\boldsymbol{u}=U_{0}(x) \hat{y}=-x \mathcal{A} \hat{y}$. The wavenumber in the $x$ direction increases as $k_{y} \mathcal{A} t$, linearly proportional to time $t$.

(Kim \& Dubrulle 2001; Kim \& Diamond 2003; Kim et al. 2004; Kim \& Diamond 2004). This shear stabilization has been recognized to be critical to regulating turbulence in many physical systems, including laboratory plasmas (Burrell 1997; Hahm 2002; Kim 2004), geophysial systems (Busse 1983), earth atmosphere (McIntyre 1989), etc. In particular, it is now thought to be the most promising mechanism to achieve an economic future reactor with a good confinement. Similar effect of a shear flow is likely to play a crucial role in the transport in the tachocline, especially by the radial shear which is thought to be stable due to stable stratification. Furthermore, as shearing is most effective in reducing the transport in the direction orthogonal to the shear flow (i.e., in the $x$ direction in Fig. 1), it can cause the anisotropy in turbulent transport as well as turbulence intensity in the tachocline.

In addition to the shearing by the radial differential rotation, Coriolis forces due to the average rotation may also quench the transport and lead to anisotropic turbulence in the tachocline. This effect of Coriolis forces has been studied in the context of the transport of angular momentum (e.g. Rüdiger 1983; Kichatinov 1987; Rüdiger 1989; Kichatinov \& Rüdiger 1993) and heat (Rüdiger 1989; Kichatinov et al. 1994; Kichatinov \& Rüdiger 1995) in the convection zone to understand the prominent latitudinal differential rotation in that region. In particular, in the limit of strong rotation such that the rotation rate exceeds the background turbulence decorrelation rate, which is the case for the Sun and most single main-sequence stars (Basri 1985), the turbulent viscosity (eddy viscosity) and heat diffusivity are shown to be reduced inversely proportional to the average rotation rate $\Omega$ while their values parallel to the rotation axis are a factor of 2 and 4 larger than those in the perpendicular directions, respectively (e.g. Kichatinov et al. 1994). Furthermore, Coriolis forces can give rise to the so-called $\Lambda$ effect (similar to the $\alpha$ effect in dynamos) from the non-diffusive part of Reynolds stress which is proportional to the rotation itself if the background turbulence in the absence of Coriolis forces is anisotropic (e.g. Rüdiger 1983) or inhomogeneous (Kichatinov 1987). In the case of strong rotation, the $\Lambda$ effect due to the anisotropy in the background turbulence also decreases as $\Omega^{-2}$ (Rüdiger 1983). Note that in these previous works, the effect of rotation shear was treated perturbatively.

The aim of this paper is to develop a self-consistent theory of turbulent transport incorporating the effect of shearing on transport, for the first time in the solar context. We focus on the shearing due to the radial different rotation in the tachocline 
and study how it affects the turbulent transport. In contrast to most of previous works which often crudely parameterized turbulent coefficients and then adjusted them to obtain agreement between prediction and observations, we shall self-consistently compute them under the physically plausible assumption that turbulence arises either from plumes penetrating from convection zone or from the instabilities of the latitudinal differential rotation. We will demonstrate that in both cases the transport can significantly be reduced by shear stabilization, with the different reduction in the horizontal plane and vertical direction. Specifically, we show that the shearing by the radial differential rotation leads to much stronger reduction in the particle transport in the radial direction, orthogonal to the shear flow (with the scaling $\propto \mathcal{A}^{-2}$ with the shearing rate $\mathcal{A}$ ) than in the horizonal plane (with the scaling $\propto \mathcal{A}^{-4 / 3}$ ). A similar, but weaker, anisotropy also develops in the amplitude of turbulent flow. In the case where the turbulence is driven by the instability of the latitudinal differential rotation, the overall amplitude of vertical particle transport as well as vertical turbulent flow becomes negligible. Special attention will be paid to the distinction between turbulence intensity and turbulent transport which have different physical origins and thus different scalings with $\mathcal{A}$, as shall be shown. These results suggest that even without density stratification, the radial differential rotation alone can give rise to anisotropic turbulent transport in the tachocline. We will also provide a self-consistent theory of the transport by a meridional flow by incorporating the effect of shear flow on turbulence, and assess its efficiency by comparing it with turbulent transport. To understand the effect of shear flows on the transport in the simplest way, the analysis in the present paper shall be limited to the case of non-magnetized tachocline with incompressible fluid, with no background density stratification, and with only radial differential shear. To keep the analysis tractable, the effect of Coriolis forces will also be ignored. While the average rotation rate is comparable to radial shear in the tachocline (i.e., $\Omega \sim \mathcal{A}$ ), the incorporation of Coriolis forces is unlikely to fundamentally change the results obtained in the paper for the following reason. Since the turbulent heat conductivity tensor considered by the previous authors (e.g. see Eq. (7) in Kichatinov et al. 1994) takes the same form as turbulent particle diffusivity tensor (in the absence of shearing), a similar quenching and anisotropy found in heat diffusivity is expected for turbulent diffusivity of particles due to Coriolis forces. Thus, the quenching in turbulent (eddy) viscosity and particle diffusivity due to Coriolis forces would become proportional to $\Omega^{-1}$, which is much weaker than the reduction $\propto \mathcal{A}^{-2}$ caused by radial shear, found in this paper. Furthermore, while Coriolis forces alone favor the transport in the direction parallel to the rotation axis, the resulting anisotropy in eddy viscosity and turbulent diffusivity will be very weak, being only a factor of 2 and 4 . In comparison, the anisotropy due to the radial shear depends on a small parameter inversely proportional to $\mathcal{A}$, and can thus become very large for strong shear $\mathcal{A}$. The effects of the magnetic fields, density stratification, and latitudinal differential rotation as well as Coriolis forces will be considered in the subsequent papers.

The remainder of the paper is organized as follows. We shall first consider the case where transport takes place due to turbulence with the negligible effect from meridional circulation and formulate the problem in Sect. 2. In Sect. 3, the effect of radial shear on turbulence intensity and momentum and particle transport will be discussed. We then include the meridional flow and investigate its effect on particle transport in Sect. 4. Section 5 contains discussions and conclusions.

\section{Formulation of the problem}

We consider incompressible fluid with no average rotation and local cartesian coordinates $x, y$, and $z$ for radial, azimuthal, and latitudinal directions, respectively. Then, main governing equations for the total velocity $\boldsymbol{u}$ and density $n$ of chemical elements are as follows:

$$
\begin{aligned}
\partial_{t} \boldsymbol{u}+\boldsymbol{u} \cdot \nabla \boldsymbol{u} & =-\nabla p+v \nabla^{2} \boldsymbol{u}+\boldsymbol{f} \\
\nabla \cdot \boldsymbol{u} & =0 \\
\left(\partial_{t}+\boldsymbol{u} \cdot \nabla\right) n & =D \nabla^{2} n .
\end{aligned}
$$

Here, $v$ and $D$ are molecular viscosity and diffusivity, respectively; $f$ in Eq. (1) is the small-scale forcing which includes the buoyancy force; the total velocity $\boldsymbol{u}=\boldsymbol{U}_{0}+\boldsymbol{v}$ includes largescale radial differential rotation $\boldsymbol{U}_{0}=U_{0}(x) \hat{y}$ and small-scale fluctuations $\boldsymbol{v}$; the total density $n=n_{0}(x)+n^{\prime}$ contains mean component $n_{0}(x)$ and fluctuating component $n^{\prime}$. Thus, the average over fluctuations of total density and velocity becomes

$\langle n\rangle=n_{0},\langle\boldsymbol{u}\rangle=\boldsymbol{U}_{0}$,

where single angular brackets \langle\rangle denote the average over fluctuations (or the statistics of the forcing).

To simplify the analysis, we shall limit ourselves to the case of unit Prandtl number $(v=D)$ and to the quasi-linear evolution (see, e.g. Moffatt 1978) of mean fields and fluctuations. By taking average of Eq. (3), we obtain the following equations for the mean fields $n_{0}$ and $U_{0}$ :

$\partial_{t} n_{0}=\partial_{x}\left[\left(D_{\mathrm{T}}^{x x}+D\right) \partial_{x} n_{0}\right]$,

$\partial_{t} U_{0}=-\partial_{y}\langle p\rangle+\partial_{x}\left[\left(v_{\mathrm{T}}^{x x}+D\right) \partial_{x} U_{0}\right]$,

where $D_{\mathrm{T}}^{x x}$ and $v_{\mathrm{T}}^{x x}$ are the vertical turbulent diffusivity and viscosity, defined by $\left\langle v_{x} n^{\prime}\right\rangle=-D_{\mathrm{T}}^{x x} \partial_{x} n_{0}$, and $\left\langle v_{x} v_{y}\right\rangle=-v_{\mathrm{T}}^{x x} \partial_{x} U_{0}$.

For the evolution of fluctuations, we approximate the radial differential rotation by a linear shear flow with $U_{0}=-x \mathcal{A}$ (here, $\mathcal{A}$ is shearing rate which is assumed to be positive without loss of generality) and obtain the following equations for $\boldsymbol{v}$ and $n^{\prime}$ from Eqs. (1)-(3):

$$
\begin{aligned}
\partial_{t} \boldsymbol{v}-x \mathcal{A} \partial_{y} \boldsymbol{v}-\mathcal{A} v_{x} \hat{y} & =-\nabla p+D \nabla^{2} \boldsymbol{v}+\boldsymbol{f} \\
\nabla \cdot \boldsymbol{v} & =0 \\
\left(\partial_{t}-x \mathcal{A} \partial_{y}\right) n^{\prime} & =-v_{x} \partial_{x} n_{0}+D \nabla^{2} n^{\prime}
\end{aligned}
$$

In the tachocline, the typical values of $D$ and $v$ are $D \sim$ $27 \mathrm{~cm}^{2} \mathrm{~s}^{-1}$ and $v \sim 10^{2} \mathrm{~cm}^{2} \mathrm{~s}^{-1}$. Thus, the assumption of the unit Prandtl number $(D=v)$ is reasonable for the solar tachocline. Furthermore, the radial differential rotation across the tachocline is $\mathcal{A} \sim 3 \times 10^{-6} \mathrm{~s}^{-1}$. Therefore, the effect of radial shear can dominate over that of dissipation with $\mathcal{A}>D k^{2}$ for $k<10^{-4} \mathrm{~cm}^{-1} \sim 10^{6} / H_{0}$, where $H_{0} \sim 6 \times 10^{9}$ is the pressure scale height. In order to capture this strong shearing effect, 
we need a method that can incorporate the effect of shearing non-perturbatively in solving Eqs. (6)-(8). To this end, we introduce the following Fourier transform for $n^{\prime}$ :

$n^{\prime}(\boldsymbol{x}, t)=\frac{1}{(2 \pi)^{3}} \int \mathrm{d}^{3} k \tilde{n}(\boldsymbol{k}, t) \exp \left\{i\left(k_{x}(t) x+k_{y} y+k_{z} z\right)\right\}$,

and similarly for $v_{i}(i=1,2,3), p$, and $f$, where $k_{x}$ satisfies an eikonal equation

$\partial_{t} k_{x}(t)=k_{y} \mathcal{A}$

Equation (10) implies that $k_{x}$ linearly increases in time as $k_{x}(t)=k_{x}(0)+k_{y} \mathcal{A} t$, explicitly reflecting the main effect of shearing by a shear flow $U_{0}(x) \hat{y}$, i.e., generation of fine scales $x$ due to tilting and distortion of fluid eddies (see Fig. 1).

With the help of Eq. (9), the solutions to Eqs. (6)-(8) can be obtained after a long, but straightforward algebra. Referring the readers to Appendix A for intermediate steps, in this section, we simply provide the solutions, which are

$$
\begin{aligned}
\tilde{v}_{x}(\boldsymbol{k}(t), t)= & \int \mathrm{d} t_{1} \mathrm{~d}^{3} k_{1} \frac{k_{y}^{2}}{k^{2}} \hat{g}\left(\boldsymbol{k}, t ; \boldsymbol{k}_{1}, t_{1}\right) \mathrm{e}^{-D Q\left(t, t_{1}\right)} \tilde{h}_{1}\left(\boldsymbol{k}_{1}, t_{1}\right), \\
\tilde{v}_{z}(\boldsymbol{k}(t), t)= & \int \mathrm{d} t_{1} \mathrm{~d}^{3} k_{1} \hat{g}\left(\boldsymbol{k}, t ; \boldsymbol{k}_{1}, t_{1}\right) \mathrm{e}^{-D Q\left(t, t_{1}\right)} \\
& \times\left[\frac{k_{y}^{2}}{k_{\mathrm{H}}^{2}} \tilde{h}_{2}\left(\boldsymbol{k}_{1}, t_{1}\right)-\left\{\frac{k_{x} k_{z} k_{y}^{2}}{k_{\mathrm{H}}^{2} k^{2}}+\frac{k_{z} k_{y}^{2}}{\left|k_{\mathrm{H}}^{3}\right|}\left[\tan ^{-1}\left(\frac{k_{x}}{\left|k_{\mathrm{H}}\right|}\right)\right.\right.\right. \\
& \left.\left.\left.-\tan ^{-1}\left(\frac{k_{1 x}}{\left|k_{1 H}\right|}\right)\right]\right\} \tilde{h}_{1}\left(\boldsymbol{k}_{1}, t_{1}\right)\right], \\
\tilde{v}_{y}(\boldsymbol{k}(t), t)= & -\frac{k_{x}}{k_{y}} \tilde{v}_{x}(\boldsymbol{k}(t), t)-\frac{k_{z}}{k_{y}} \tilde{v}_{z}(\boldsymbol{k}(t), t), \\
\tilde{n}(\boldsymbol{k}(t), t)= & -\partial_{x} n_{0} \int \mathrm{d} t_{1} \mathrm{~d}^{3} k_{1} \hat{g}\left(\boldsymbol{k}, t ; \boldsymbol{k}_{1}, t_{1}\right) \mathrm{e}^{-D Q\left(t, t_{1}\right)} \tilde{v}_{x}\left(\boldsymbol{k}_{1}, t_{1}\right) .(1
\end{aligned}
$$

Here, $Q\left(t, t_{1}\right)=\int_{t_{1}}^{t} \mathrm{~d} t^{\prime}\left[k_{x}^{2}\left(t^{\prime}\right)+k_{\mathrm{H}}^{2}\right]=\left[k_{x}^{3}-k_{1 x}^{3}\right] / 3 k_{y} \mathcal{A}+k_{\mathrm{H}}^{2}\left(t-t_{1}\right)$; $k_{\mathrm{H}}^{2}=k_{y}^{2}+k_{z}^{2}$ is the amplitude of wave number in the horizontal plane; $k^{2}=k_{\mathrm{H}}^{2}+k_{x}^{2} ; \hat{g}$ is the Green's function given by

$$
\begin{aligned}
\hat{g}\left(\boldsymbol{k}, t ; \boldsymbol{k}_{1}, t_{1}\right)= & \delta\left(k_{y}-k_{1 y}\right) \delta\left(k_{z}-k_{1 z}\right) \\
& \times \delta\left[k_{x}-k_{1 x}-k_{1 y}\left(t-t_{1}\right) \mathcal{A}\right] ;
\end{aligned}
$$

$\tilde{h}_{1}$ and $\tilde{h}_{2}$ are the forcing terms which are related to $\tilde{f}_{i}$ as

$\tilde{h}_{1}=\left(1+k_{z}^{2} / k_{y}^{2}\right) \tilde{f}_{x}-k_{x} \tilde{f}_{y} / k_{y}-k_{x} k_{z} \tilde{f}_{z} / k_{y}^{2}$

$\tilde{h}_{2}=-k_{z} \tilde{f}_{y} / k_{y}+\tilde{f}_{z}$.

In the following sections, turbulence amplitude $\left\langle v_{x}^{2}\right\rangle$ and $\left\langle v_{z}^{2}\right\rangle$, particle flux $\left\langle n^{\prime} v_{x}\right\rangle=-D_{\mathrm{T}} \partial_{x} n_{0}$, and momentum flux $\left\langle v_{x} v_{y}\right\rangle=$ $-v_{\mathrm{T}}^{x x} \partial_{x} U_{0}$, will be computed. For simplicity, we assume spatially homogeneous and temporally short correlated forcing $\boldsymbol{f}$, which is incompressible $(\nabla \cdot f=0)$ and reflectionally symmetric. Specifically, the forcing correlation functions are taken to be of the following form:

$\left\langle\tilde{f}_{i}\left(\boldsymbol{k}_{1}, t_{1}\right) \tilde{f}_{j}\left(\boldsymbol{k}_{2}, t_{2}\right)\right\rangle=\tau_{f}(2 \pi)^{3} \delta\left(\boldsymbol{k}_{1}+\boldsymbol{k}_{2}\right) \psi_{i j}\left(\boldsymbol{k}_{2}\right)$,

where $\tau_{f}$ is the (short) correlation time of the forcing.

\section{Turbulence amplitude and transport}

In this section, we shall demonstrate that the radial shear reduces turbulence amplitude $\left\langle v_{x}^{2}\right\rangle$ in the vertical direction and $\left\langle v_{z}^{2}\right\rangle$ in the horizontal plane, and vertical transport of momentum and particles by shear stabilization. The degree of reduction by shearing can be different in each case; in particular, turbulence amplitude depends on the direction, suggesting anisotropic turbulence.

\subsection{Turbulence amplitude}

From Eqs. (9)-(12) and (15)-(18), we obtain the following:

$$
\begin{aligned}
\left\langle v_{x}^{2}\right\rangle= & \frac{\tau_{f}}{(2 \pi)^{3}} \int \mathrm{d}^{3} k \mathrm{~d} t_{1} \mathrm{~d}^{3} k_{1} \frac{k_{y}^{4}}{k^{4}} \mathrm{e}^{-2 D Q\left(t, t_{1}\right)} \\
& \times \hat{g}\left(\boldsymbol{k}, t ; \boldsymbol{k}_{1}, t_{1}\right) \phi_{11}\left(\boldsymbol{k}_{1}\right) \\
\left\langle v_{z}^{2}\right\rangle= & \frac{\tau_{f}}{(2 \pi)^{3}} \int \mathrm{d}^{3} k \mathrm{~d} t_{1} \mathrm{~d}^{3} k_{1} \mathrm{e}^{-2 D Q\left(t, t_{1}\right)} \hat{g}\left(\boldsymbol{k}, t ; \boldsymbol{k}_{1}, t_{1}\right)\left\{\frac{k_{y}^{4}}{k_{\mathrm{H}}^{4}} \phi_{22}\left(\boldsymbol{k}_{1}\right)\right. \\
& -2 \frac{k_{y}^{2}}{k_{\mathrm{H}}^{2}}\left[\frac { k _ { y } ^ { 2 } k _ { z } } { k _ { \mathrm { H } } ^ { 3 } } \left(\tan ^{-1}\left(\frac{k_{x}}{\left|k_{\mathrm{H}}\right|}\right)\right.\right. \\
& \left.\left.-\tan ^{-1}\left(\frac{k_{1 x}}{\left|k_{1 H}\right|}\right)\right)+\frac{k_{y}^{2} k_{z} k_{x}}{k_{\mathrm{H}}^{2} k^{2}}\right] \phi_{12}\left(\boldsymbol{k}_{1}\right) \\
& +\left[\frac { k _ { y } ^ { 2 } k _ { z } } { k _ { \mathrm { H } } ^ { 3 } } \left(\tan ^{-1}\left(\frac{k_{x}}{\left|k_{\mathrm{H}}\right|}\right)\right.\right. \\
& \left.\left.\left.-\tan ^{-1}\left(\frac{k_{1 x}}{\left|k_{1 H}\right|}\right)\right)+\frac{k_{y}^{2} k_{z} k_{x}}{k_{\mathrm{H}}^{2} k^{2}}\right]^{2} \phi_{11}\left(\boldsymbol{k}_{1}\right)\right\} .
\end{aligned}
$$

Here, $\phi_{i j}(\boldsymbol{k})(i=1,2)$ are the power spectra of the forcing defined by:

$\left\langle\tilde{h}_{i}\left(\boldsymbol{k}_{1}, t_{1}\right) \tilde{h}_{j}\left(\boldsymbol{k}_{2}, t_{2}\right)\right\rangle=\tau_{f}(2 \pi)^{3} \delta\left(\boldsymbol{k}_{1}+\boldsymbol{k}_{2}\right) \phi_{i j}\left(\boldsymbol{k}_{2}\right)$

with the (short) forcing correlation time $\tau_{f}$. Recall that $Q\left(t, t_{1}\right)=\left[k_{x}^{3}-k_{1 x}^{3}\right] / 3 k_{y} \mathcal{A}+k_{\mathrm{H}}^{2}\left(t-t_{1}\right), k_{\mathrm{H}}^{2}=k_{y}^{2}+k_{z}^{2}$, and $k^{2}=k_{\mathrm{H}}^{2}+k_{x}^{2}$ and that $\tilde{h}_{i}\left(\boldsymbol{k}_{1}, t_{1}\right)$ (for $i=1,2$ ) is given by Eqs. (16)-(17). For an incompressible forcing, one can easily show that $\phi_{i j}$ in Eq. (21) is related to $\psi_{i j}$, defined in Eq. (18), as

$$
\begin{aligned}
& \phi_{11}=\frac{k^{4}}{k_{y}^{4}} \psi_{11}, \phi_{12}=\frac{1}{k_{y}^{4}}\left(k^{2} k_{x} k_{z} \psi_{11}+k^{2} k_{\mathrm{H}}^{2} \psi_{13}\right), \\
& \phi_{22}=\frac{1}{k_{y}^{4}}\left(k_{x}^{2} k_{z}^{2} \psi_{11}+k_{\mathrm{H}}^{4} \psi_{33}+2 k_{x} k_{z} k_{\mathrm{H}}^{2} \psi_{13}\right) .
\end{aligned}
$$

As we are interested in the shearing effect, and also as it is likely that $\mathcal{A} \gg D k_{y}^{2}$ in the tachocline, we evaluate the integrals in Eqs. (19)-(20) in the strong shear limit $\xi \equiv D k_{y}^{2} / \mathcal{A} \ll 1$. Note here that $k_{y}$ is the inverse of the characteristic scale of the forcing in the $y$ (azimuthal) direction. To this end, we express the integral over $t_{1}$ in terms of a new time variable $\tau=k_{x} / k_{y}=$ $k_{1 x} / k_{1 y}+\mathcal{A}\left(t-t_{1}\right)$. Thus, $D Q\left(t, t_{1}\right)=\xi\left[\tau^{3} / 3+\gamma \tau-\left(a^{3} / 3+\gamma a\right)\right]$, where $\gamma=k_{\mathrm{H}}^{2} / k_{y}^{2}$ and $a=k_{1 x} / k_{1 y}$. Then, by taking the limit 
$\xi \rightarrow 0$ in the $\tau$ integral, and by assuming $\psi_{i j}\left(k_{y}\right)=\psi_{i j}\left(-k_{y}\right)$, we obtain

$$
\begin{aligned}
\left\langle v_{x}^{2}\right\rangle \sim & \frac{\tau_{f}}{2(2 \pi)^{3} \mathcal{A}} \int \mathrm{d}^{3} k\left(\frac{k_{y}^{2}}{k_{\mathrm{H}}^{2}}\right)^{3 / 2} \\
& \times\left[\frac{\pi}{2}-\tan ^{-1}(a / \sqrt{\gamma})+\frac{\sqrt{\gamma} a}{\gamma+a^{2}}\right] \phi_{11}(\boldsymbol{k}), \\
\left\langle v_{z}^{2}\right\rangle \sim & \frac{\tau_{f}}{(2 \pi)^{3} \mathcal{A}} \int \mathrm{d}^{3} k \frac{1}{3} \Gamma\left(\frac{1}{3}\right)\left(\frac{3 \mathcal{A}}{2 D k_{y}^{2}}\right)^{1 / 3} \frac{k_{y}^{4}}{k_{\mathrm{H}}^{4}} \\
& \times\left[\phi_{22}(\boldsymbol{k})+\left(\frac{\pi}{2}-\tan ^{-1}(a / \sqrt{\gamma})\right)^{2} \frac{k_{z}^{2}}{k_{\mathrm{H}}^{2}} \phi_{11}(\boldsymbol{k})\right],
\end{aligned}
$$

to leading order in $\xi \ll 1$. Here, $\gamma=k_{\mathrm{H}}^{2} / k_{y}^{2}, a=k_{x} / k_{y}$, and $\Gamma(x)$ is Gamma function. Equations (23) and (24) reveal that turbulence amplitude in both horizontal and vertical directions is reduced as shearing rate $\mathcal{A}$ increases, with the scaling $\left\langle v_{x}^{2}\right\rangle \propto \mathcal{A}^{-1}$ and $\left\langle v_{z}^{2}\right\rangle \propto \mathcal{A}^{-1}\left(\mathcal{A} / D k_{y}^{2}\right)^{1 / 3}$. Recall that $k_{y}$ is the inverse of the characteristic scale of the forcing in the $y$ (azimuthal) direction. Since these results were obtained in the strong shear limit where $\mathcal{A} / D k_{y}^{2} \gg 1,\left\langle v_{z}^{2}\right\rangle \gg\left\langle v_{x}^{2}\right\rangle$. For instance, for the typical values of parameters in the tachocline, $D \sim 30 \mathrm{~cm}^{2} \mathrm{~s}^{-1}$ and $\mathcal{A} \sim 3 \times 10^{-6} \mathrm{~s}^{-1}, \mathcal{A} / D k_{y}^{2} \sim 10^{6}$ with $\left\langle v_{z}^{2}\right\rangle \sim 10^{2}\left\langle v_{x}^{2}\right\rangle$ if $k_{y} \sim 10^{-7} \mathrm{~cm}^{-1} \sim 10^{3} / H_{0}$ while $\mathcal{A} / D k_{y}^{2} \sim 10^{11}$ with $\left\langle v_{z}^{2}\right\rangle \sim 10^{4}\left\langle v_{x}^{2}\right\rangle$ if $k_{y} \sim 10^{-9} \mathrm{~cm}^{-1} \sim 10 / H_{0}$.

Therefore, the reduction in turbulence amplitude by the radial shear is more severe in the radial direction than in the horizontal plane, with the anisotropy in turbulence amplitude. This is because a shear flow in the $y$ direction, varying in $x$, shears the radial turbulent flow $v_{x}$ directly while its shearing only indirectly influences the horizontal flow $v_{z}$ through the incompressible condition $(\nabla \cdot v=0)$ and enhanced dissipation for finite $D$. Thus, shearing regularizes the horizontal turbulence amplitude $\left\langle v_{x}^{2}\right\rangle$, with its value remaining finite even in the absence of dissipation $(D=0)$. In contrast, it is a finite dissipation $(D \neq 0)$ that keeps $\left\langle v_{z}^{2}\right\rangle$ finite. The anisotropy in turbulence amplitude should, however, be distinguished from that in turbulent transport, as will be discussed in Sect. 4. Equations (23)-(24) also indicate that the precise value of $\left\langle v_{x}^{2}\right\rangle /\left\langle v_{z}^{2}\right\rangle$ depends on the characteristics of the forcing, such as power spectrum $\phi_{i j}(\boldsymbol{k})$ and typical wavenumber $\boldsymbol{k}$. We briefly discuss this dependence in the following.

If the turbulence is mainly driven from plumes penetrating from the convection zone (see Fig. 2), the power spectrum is likely to be anisotropic with $\left|k_{x}\right| \ll\left|k_{y}\right| \sim\left|k_{z}\right|$. Thus, $\phi_{11} \sim \psi_{11}$ and $\phi_{22} \sim 0$ with $\left\langle v_{x}^{2}\right\rangle /\left\langle v_{z}^{2}\right\rangle \sim\left(\mathcal{A} / D k_{y}^{2}\right)^{-1 / 3} \ll 1$. For example, for the parameter values of $D \sim 30 \mathrm{~cm}^{2} \mathrm{~s}^{-1}, \mathcal{A} \sim 3 \times 10^{-6} \mathrm{~s}^{-1}$, and $k_{y} \sim 10^{-7} \mathrm{~cm}^{-1} \sim 10^{3} / H_{0},\left\langle v_{x}^{2}\right\rangle /\left\langle v_{z}^{2}\right\rangle \sim 10^{-2}$. In contrast, if turbulence is due to the instability of latitudinal differential rotation, the forcing is likely act only on the horizontal plane with $f_{x} \sim 0$. Thus, $\psi_{11} \sim 0$, rendering $\phi_{11} \sim 0$ and $\phi_{22} \sim \psi_{33}$ in Eq. (22). Thus, in this case, there is no turbulence in the radial direction with $\left\langle v_{x}^{2}\right\rangle \sim 0$ while the horizontal turbulent flow is reduced for large $\mathcal{A}$ as $\left\langle v_{z}^{2}\right\rangle \propto \mathcal{A}^{-1}\left(\mathcal{A} / D k_{y}^{2}\right)^{1 / 3}$. This result of the reduction in the horizontal turbulence (parallel to the shear flow) in the absence of vertical flows, which may seem surprising to some readers, is basically due to the enhanced

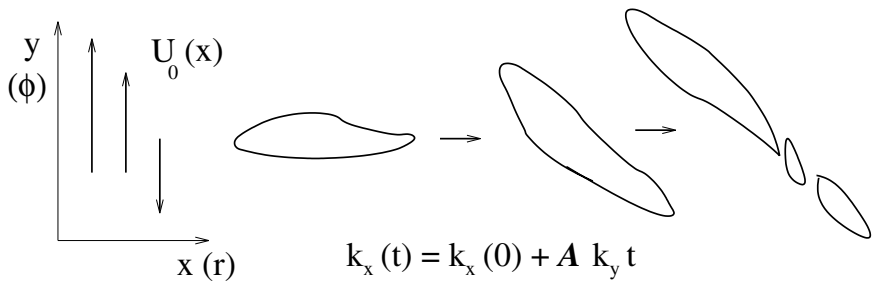

Fig. 2. Tilting and break-up of turbulent eddy driven by plumes due to the shearing by radial differential rotation $\boldsymbol{u}=U_{0}(x) \hat{y}=-\mathcal{A} x \hat{y}$. The wavenumber in the $x$ direction again increases as $k_{y} \mathcal{A} t$, linearly proportional to time $t$. The turbulent eddy is almost uniform in $x$ with $k_{x} / k_{y} \ll 1$ initially at $t=0$, but gradually develops radial dependences as the shearing continues.

dissipation by shearing for $D \neq 0$, as noted previously. That is, even if the forcing drives only horizontal turbulent flows, the shear flow creates fine scales in the vertical direction in these flows, enhancing their dissipation rate. Therefore, in both cases, the turbulence amplitude in the radial direction is always much weaker than that in the horizontal plane (see Fig. 2). It is also interesting to consider the special case of an isotropic forcing where $\psi_{i j}(\boldsymbol{k})=\left[\delta_{i j}-k_{i} k_{j} / k^{2}\right] F(k)$, with

$\phi_{11}=k^{2} k_{\mathrm{H}}^{2} F(k) / k_{y}^{4}, \phi_{12}=0, \phi_{22}=k_{\mathrm{H}}^{2} F(k) / k_{y}^{2}$.

Even in this case, the anisotropy again appears in the turbulence amplitude as $\left\langle v_{x}^{2}\right\rangle /\left\langle v_{z}^{2}\right\rangle \sim\left(\mathcal{A} / D k_{y}^{2}\right)^{-1 / 3} \ll 1$ (see Fig. 1). Therefore, in all possible cases, shearing by radial differential rotation is very likely to lead to turbulence which is stronger in the horizontal plane than in the vertical direction (see Figs. 1 and 2).

\subsection{Turbulent transport}

The amplitudes of turbulent flow $\left\langle v_{x}^{2}\right\rangle$ and $\left\langle v_{z}^{2}\right\rangle$ are often used to characterize turbulent transport in the $x$ and $z$ direction. However, turbulence amplitude and turbulent transport are not simply related to each other since they have different physical origins. In particular, the irreversibility is necessary for turbulent transport, but not for turbulence amplitude (see, e.g., Kim $\&$ Diamond 2003). Furthermore, the transport of momentum in general differs from the transport of particles. In the following, we will demonstrate that this is indeed the case.

In order to compute momentum and particle fluxes (thereby turbulent viscosity $v_{\mathrm{T}}^{x x}$ and diffusivity $D_{\mathrm{T}}^{x x}$, we go through a similar procedure by using Eqs. (9)-(13), (15)-(17), and (21) (see Appendix B for details). The results are

$$
\begin{aligned}
v_{\mathrm{T}}^{x x} \sim & \frac{\tau_{f}}{2(2 \pi)^{3} \mathcal{A}^{2}} \int \mathrm{d}^{3} k \phi_{11}(\boldsymbol{k}) \frac{k_{y}^{2}}{k_{\mathrm{H}}^{2}} \\
& \times\left[-\frac{k_{y}^{2}}{k^{2}}+\frac{k_{z}^{2}}{k_{\mathrm{H}}^{2}}\left(\frac{\pi}{2}-\tan ^{-1}(a / \sqrt{\gamma})\right)^{2}\right], \\
D_{\mathrm{T}}^{x x} \sim & \frac{\tau_{f}}{2(2 \pi)^{3} \mathcal{A}^{2}} \int \mathrm{d}^{3} k \phi_{11}(\boldsymbol{k}) \frac{k_{y}^{2}}{k_{\mathrm{H}}^{2}}\left[\frac{\pi}{2}-\tan ^{-1}(a / \sqrt{\gamma})\right]^{2},
\end{aligned}
$$

to leading order in $\xi \ll 1$, with $\gamma=k_{\mathrm{H}}^{2} / k_{y}^{2}$ and $a=k_{x} / k_{y}$. Equations (26) and (27) clearly illustrate the strong reduction 
in momentum and particle transport due to shearing effect, with the scaling $v_{\mathrm{T}}^{x x} \propto \mathcal{A}^{-2}$ and $D_{\mathrm{T}}^{x x} \propto \mathcal{A}^{-2}$. The precise value of their amplitudes, however, depend on the properties of the forcing. For instance, in the case where the forcing is due to the instability of the latitudinal differential rotation with $f_{x}=0$ (i.e., $\phi_{11}=0$ ), there is no vertical transport with $D_{\mathrm{T}}^{x x}=v_{\mathrm{T}}^{x x}=0$, as expected. Besides this extreme case, $D_{\mathrm{T}}^{x x}$ and $v_{\mathrm{T}}^{x x}$ seem to have similar amplitude of the same order of magnitude. That is, within this hydrodynamic turbulence model, particle transport is comparable to momentum transport.

Let us examine, in some detail, other important implications of Eqs. (26) and (27), excluding the case where $f_{x}=0$. First, Eq. (26) shows that turbulent viscosity can be either positive or negative depending on characteristic scales of the forcing. In the 2D limit where $k_{z} \rightarrow 0$ (i.e. in the $x-y$ plane), $v_{\mathrm{T}}^{x x}$ becomes negative, recovering the well-known result of inverse cascade in 2D hydrodynamic turbulence due to the conservation of enstropy (see, e.g., Eq. (33) in Kim \& Dubrulle 2001). Negative viscosity means that turbulence acts as a source of large-scale shear flow. Equation (26) clearly shows how this negative viscosity becomes positive in 3D hydrodynamic turbulence for $k_{z} \gtrsim k_{y}$, with turbulence now damping the shear flow. Interestingly, the result $v_{\mathrm{T}}^{x x} \sim \mathcal{A}^{-2}$ leads to a non-linear equation for $\boldsymbol{U}_{0}$ in Eq. (5), with the possibility of causing time-dependence in the evolution of the mean flow $\boldsymbol{U}_{0}$. Note that time-dependence of the radial differential rotation can also be caused by the interaction with gravity waves (Kim \& MacGregor 2001), with time variability being a generic feature of a nonlinear system. Furthermore, since turbulence (with $v_{\mathrm{T}}^{x x}>0$ ) becomes inefficient in smoothing out the profile of $\boldsymbol{U}_{0}$ as it develops larger gradient, the (angular) momentum transport by turbulence within this hydrodynamical turbulence does not seem to be easily reconciled with the apparent uniform rotation in the solar radiative interior.

Secondly, Eq. (27) shows that turbulent diffusivity $D_{\mathrm{T}}^{x x} \sim$ $\mathcal{A}^{-1}\left\langle v_{x}^{2}\right\rangle$ is proportional to $\left\langle v_{x}^{2}\right\rangle$, but with the proportionality $\mathcal{A}^{-1}$, depending on $\mathcal{A}$. That is, particle transport is not exactly proportional to turbulence amplitude, as has often been assumed. If turbulent flow has the characteristic velocity $v_{x}$ with characteristic length scale $l_{x}$ and correlation time $\tau_{x}$ in the $x$ direction, one would expect that $D_{\mathrm{T}}^{x x} \sim v_{x} l_{x} \sim \tau_{x} v_{x}^{2}$. The comparison with our result $D_{\mathrm{T}}^{x x} \sim \mathcal{A}^{-1} v_{x}^{2}$ then implies that $l_{x} \sim \mathcal{A}^{-1} v_{x}$ and $\tau_{x} \sim \mathcal{A}^{-1}$. That is, the characteristic length scale and correlation time of the turbulent flow are set by shearing time $\mathcal{A}^{-1}$, both of them becoming shorter as $\mathcal{A}$ increases. This is a clear manifestation that the decorrelation rate of two near-by points in the fluid is enhanced by a shear flow (the socalled enhanced decorrelation) with a shorter correlation length (Biglari et al. 1990), as a result of tilting and distortion of eddies. This point will again be emphasized in Sect. IV in considering particle transport in the horizontal plane $\left(D_{\mathrm{T}}^{z z}\right)$.

Finally, we estimate the minimum velocity of forcing (e.g., the velocity of plumes) in order for the turbulent transport $D_{\mathrm{T}}^{x x}$ to be at least larger than molecular value $D \sim 30 \mathrm{~cm}^{2} \mathrm{~s}^{-1}$. To this end, we approximate Eq. (27) as

$$
D_{\mathrm{T}}^{x x} \sim \frac{\tau_{f}\left\langle f^{2}\right\rangle}{\mathcal{A}^{2}}
$$

where $\left\langle f^{2}\right\rangle \sim \int \mathrm{d}^{3} k \psi_{i i}(\boldsymbol{k}) /(2 \pi)^{3}$ is the amplitude of the forcing. We then let $\left\langle f^{2}\right\rangle \sim v_{f}^{2} / \tau_{f}^{2}$ with a typical amplitude of forcing velocity $v_{f}$ and take $\mathcal{A} \sim 3 \times 10^{-6} \mathrm{~s}^{-1}$ and $\tau_{f} \mathcal{A} \sim 10^{-2}$. Then, $D_{\mathrm{T}}^{x x} \sim D \sim 30 \mathrm{~cm}^{2} \mathrm{~s}^{-1}$ (molecular diffusivity) gives the minimum value of $v_{f} \sim 10^{-3} \mathrm{~cm} \mathrm{~s}^{-1}$. The minimum value of the vertical turbulent velocity can easily be found from $\left\langle v_{x}^{2}\right\rangle \sim \mathcal{A} D_{\mathrm{T}}^{x x}$ with $D_{\mathrm{T}}^{x x}=D$ to be $v_{x} \sim 10^{-2} \mathrm{~cm} \mathrm{~s}^{-1}$. In this case, the horizontal turbulent velocity becomes $v_{z} \sim 0.1 \mathrm{~cm} \mathrm{~s}^{-1}$ for $k_{y} \sim 10 / H_{0}$ while $v_{z} \sim 1 \mathrm{~cm} \mathrm{~s}^{-1}$ for $k_{y} \sim 10^{3} / H_{0}$. Therefore, plumes with a reasonably modest velocity $v_{f}>10^{-3} \mathrm{~cm} \mathrm{~s}^{-1}$ are likely to enhance vertical diffusivity of particles over the molecular value. However, if these plumes are rarely occurring with a very small filling factor (Brummell et al. 2002; Rempel 2004), it is more reasonable to take $\left\langle f^{2}\right\rangle \sim \alpha v_{f}^{2} / \tau_{f}^{2}$ with a filling factor $\alpha$ and typical plume velocity $v_{f}$. Thus, Eq. (28) becomes

$D_{\mathrm{T}}^{x x} \sim \frac{\alpha v_{f}^{2}}{\tau_{f} \mathcal{A}^{2}}$.

For instance, for $\alpha \sim 10^{-8}$, the minimum velocity of plume for $D_{\mathrm{T}}^{x x}>D$ is increased to $v_{f} \sim 10 \mathrm{~cm} \mathrm{~s}^{-1}$, which is still modest. Thus, turbulence due to plume penetration can significantly enhance the total diffusivity of light elements over the molecular value. In arriving at this conclusion, we have, however, implicitly assumed that plumes penetrate the entire layer of the tachocline, which is unlikely to be the case in the Sun (e.g., Brummell et al. 2002). To be precise, the filling factor $\alpha$ should refer to those only which do penetrate the entire layer. The probability of such events of large amplitude will of course be small. We will discuss this point further in Sect. 4, where we study the transport of particles by a meridional circulation and its implications for mixing of light elements. Note that in the absence of plumes, other mechanisms (such as gravity waves) could mediate transport (see, e.g. García Lopez \& Spruit 1991).

\section{Effect of meridional circulation}

So far we have focused on the effect of shearing by the radial differential rotation on turbulent transport and studied how much reduction it causes in turbulence amplitude and vertical transport of particle and momentum. In this section, we will examine how the particle transport by meridional circulation is affected by the radial differential rotation, assess its efficiency compared to vertical turbulent transport of particles, and discuss the anisotropic transport of particles by computing horizontal turbulent transport of particles. As noted previously, the meridional circulation can be crucial to explaining the depletion of light elements in the Sun or massive stars.

We assume the meridional circulation to take the form $\boldsymbol{U}_{m}=\nabla \times \psi(x, z) \hat{y}=\left(-\partial_{z} \psi, 0, \partial_{x} \psi\right)$ and include it in the total velocity $\boldsymbol{u}=\boldsymbol{U}_{0}+\boldsymbol{U}_{m}+\boldsymbol{v}$. The density perturbation $n_{m}(x, z)$ due to meridional flow also contributes to the total density $n=n_{0}+n_{m}(x, z)+n^{\prime}$. We denote the average over fluctuations of total density and velocity by single angular brackets \langle\rangle as before. That is

$\langle n\rangle=n_{0}(x)+n_{m}(x, z),\langle\boldsymbol{u}\rangle=\boldsymbol{U}_{0}(x)+\boldsymbol{U}_{m}(x, z)$. 
To obtain the mean component $n_{0}$ and $U_{0}$, we introduce the average over $z$ in addition to that over fluctuations, denoting it by double angular brackets:

$\langle\langle n\rangle\rangle=n_{0}(x),\langle\langle\boldsymbol{u}\rangle\rangle=\boldsymbol{U}_{0}(x)$.

Meridinal flow $\boldsymbol{U}_{m}$, together with $n_{m}$, will lead to effective transport of $n_{0}$ with the effective diffusivity $D_{\text {eff }}$ as $\left\langle\left\langle U_{m x} n_{m}\right\rangle\right\rangle=$ $-D_{\text {eff }} \partial_{x} n_{0}$. On the other hand, the turbulence will transport $n_{m}$, as well as $n_{0}$, with non-vanishing horizontal component of turbulent diffusivity $D_{\mathrm{T}}^{z z}$. That is, $\left\langle v_{i} n^{\prime}\right\rangle=-D_{\mathrm{T}}^{i j} \partial_{j}\left(n_{0}+n_{m}\right)$. Note that the interaction between $U_{0}(x) \hat{y}$ and $\boldsymbol{U}_{m}(x, z)$ will generate the zonal component $\delta U_{0}(x, z)$ depending on $z$, which will in turn lead to non-trivial transport of $U_{0}$ due to the interaction with $\boldsymbol{U}_{m}$. However, this momentum transport by a meridional flow turns out to have a similar behaviour to particle transport, not particularly illuminating from the point of angular momentum transport in the Sun which must be much more efficient than particle transport. Furthermore, for the evolution of density, this perturbation $\delta U_{0} \hat{y}$ can be ignored. For this reason, the momentum transport by a meridional circulation will not be elaborated in this paper.

\subsection{Effective particle transport by meridional circulation}

In order to compute $D_{\text {eff }}$, we first obtain the equation for $n_{m}$ by taking the average of Eq. (3) over fluctuations with the result:

$\left[\partial_{t}+\boldsymbol{U}_{m} \cdot \nabla\right]\left(n_{0}+n_{m}\right)=\partial_{i}\left[\left(D_{\mathrm{T}}^{i j}+D \delta_{i j}\right) \partial_{j}\left(n_{0}+n_{m}\right)\right]$.

The average of Eq. (30) over $z$ then gives us the equation for $n_{0}$ as follows:

$\partial_{t} n_{0}=\partial_{x}\left[\left(D_{\mathrm{eff}}+D_{\mathrm{T}}^{x x}+D\right) \partial_{x} n_{0}\right]$,

with the effective diffusivity $D_{\text {eff }}$ due to meridional flow $\left(\left\langle\left\langle U_{m x} n_{m}\right\rangle\right\rangle=-D_{\text {eff }} \partial_{x} n_{0}\right)$. A simple equation for $n_{m}$ follows by subtracting Eq. (31) from (30), and then by assuming that $\left|\boldsymbol{U}_{m} \cdot \nabla n_{0}\right| \gg\left|\boldsymbol{U}_{m} \cdot \nabla n_{m}\right|$ :

$\partial_{t} n_{m}=-U_{m x} \partial_{x} n_{0}+\partial_{i}\left[\left(D_{\mathrm{T}}^{i j}+D \delta_{i j}\right) \partial_{j} n_{m}\right]$.

For simplicity, we assume a linear mean density profile $n_{0} \propto$ $x$ and a steady meridional circulation with $\boldsymbol{U}_{m}=U_{m}(z) \hat{x}=$ $\left(2 V_{m}\right) \cos (p z) \hat{x}$. In this case, Eq. (32) can readily be solved with the solution for $n_{m}$ as $n_{m}=-2 V_{m}\left(\partial_{x} n_{0}\right) \cos (p z) /\left(D_{\mathrm{T}}^{z z}+D\right) p^{2}$. Then, by computing the average of $U_{m} n_{m}$ over $z$, we obtain the effective diffusivity $D_{\text {eff }}$ for the mean density $n_{0}$ :

$D_{\text {eff }}=\frac{2 V_{m}^{2}}{\left(D+D_{\mathrm{T}}^{z z}\right) p^{2}} \sim \frac{2 V_{m}^{2}}{D_{\mathrm{T}}^{z z} p^{2}}$,

for $D_{\mathrm{T}}^{z z} \gg D$. Thus, the total vertical diffusivity $D_{t o t}=D_{\text {eff }}+$ $D_{\mathrm{T}}^{z z}+D$ of the mean density $n_{0}$ becomes

$D_{t o t}=\frac{2 V_{m}^{2}}{D_{\mathrm{T}}^{z z} p^{2}}+D_{\mathrm{T}}^{x x}+D$,

where $D_{\mathrm{T}}^{x x}$ is given in Eq. (27) (or Eq. (31)). Eq. (33), showing that $D_{\text {eff }}$ is inversely proportional to horizontal (particle) turbulent diffusivity, demonstrates that strong horizontal turbulence (to be precise, horizontal particle transport) with a large value of $D_{\mathrm{T}}^{z z}$ works against the transport by meridional circulation. Similar result was obtained previously by Chaboyer \& Zahn (1990). In contrast to most of the previous works which crudely parameterized $D_{\mathrm{T}}^{z z}$, we will compute it self-consistently in Sect. 4.2, by taking into account shearing effect. We will then estimate $D_{\text {eff }}$ and compare it with $D_{\mathrm{T}}^{x x}$ in Sect. 4.3

\subsection{Horizontal turbulent transport of particles}

We consider a quasi-linear equation for $n^{\prime}$ as before, but including $n_{m}$. Eq. (8) thus takes the following form:

$\left[\partial_{t}+U_{0}(x) \partial_{y}+U_{m x} \partial_{x}\right] n^{\prime}=-v_{i} \partial_{i}\left(n_{0}+n_{m}\right)+D \nabla^{2} n^{\prime}$.

Since $U_{m x}$ is very likely much weaker than $U_{0}(=-x \mathcal{A})$, we ignore $U_{m x}$ in Eq. (35) and obtain the solution to $\tilde{n}$ as

$$
\begin{aligned}
\tilde{n}(\boldsymbol{k}(t), t)= & -\left[\partial_{i}\left(n_{0}+n_{m}\right)\right] \\
& \times \int \mathrm{d} t_{1} \mathrm{~d}^{3} k_{1} \hat{g}\left(\boldsymbol{k}, t ; \boldsymbol{k}_{1}, t_{1}\right) \mathrm{e}^{-D Q\left(t, t_{1}\right)} \tilde{v}_{i}\left(\boldsymbol{k}_{1}, x, t_{1}\right),
\end{aligned}
$$

which should be compared to Eq. (14). Here, $Q\left(t, t_{1}\right)=$ $\int_{t_{1}}^{t} \mathrm{~d} t^{\prime}\left[k_{x}^{2}\left(t^{\prime}\right)+k_{\mathrm{H}}^{2}\right]=\left[k_{x}^{3}-k_{1 x}^{3}\right] / 3 k_{y} \mathcal{A}+k_{\mathrm{H}}^{2}\left(t-t_{1}\right)$. Thus, by using Eqs. (9)-(12), (15)- (17), and (21), and by a similar analysis as done before, we can obtain $D_{\mathrm{T}}^{z z}$ by computing $\left\langle n^{\prime} v_{z}\right\rangle=$ $-D_{\mathrm{T}}^{z z} \partial_{z} n_{m}$ :

$$
\begin{aligned}
D_{\mathrm{T}}^{z z} \sim & \frac{\tau_{f}}{(2 \pi)^{3} \mathcal{A}^{2}} \int \mathrm{d}^{3} k \frac{1}{3} \Gamma\left(\frac{2}{3}\right)\left(\frac{3 \mathcal{A}}{2 D k_{y}^{2}}\right)^{2 / 3} \frac{k_{y}^{4}}{k_{\mathrm{H}}^{4}} \\
& \times\left[\phi_{22}(\boldsymbol{k})+\frac{k_{z}^{2}}{k_{\mathrm{H}}^{2}}\left[\frac{\pi}{2}-\tan ^{-1}(a / \sqrt{\gamma})\right]^{2} \phi_{11}(\boldsymbol{k})\right],
\end{aligned}
$$

in the strong shear limit $\xi=D k_{y}^{2} / \mathcal{A} \ll 1$. Here, again $\gamma=k_{\mathrm{H}}^{2} / k_{y}^{2}$ and $a=k_{x} / k_{y}$. Note that the off-diagonal terms $D_{\mathrm{T}}^{i j}$ for $i \neq j$ vanish for $\psi_{i j}\left(k_{y}\right)=\psi_{i j}\left(-k_{y}\right)$ and $\psi_{i j}\left(k_{z}\right)=\psi_{i j}\left(-k_{z}\right)$. Eq. (37) demonstrates strong reduction in particle transport in horizontal plane due to shearing, with the scaling $D_{\mathrm{T}}^{z z} \propto$ $\mathcal{A}^{-2}\left(\mathcal{A} / D k_{y}^{2}\right)^{2 / 3} \propto \mathcal{A}^{-4 / 3}$. However, this reduction is less severe than that in the radial direction, which is $D_{\mathrm{T}}^{x x} \propto \mathcal{A}^{-2}$ (see Eq. (27)). That is, $D_{\mathrm{T}}^{x x} / D_{\mathrm{T}}^{z z} \propto\left(\mathcal{A} / D k_{y}^{2}\right)^{-2 / 3}$. For instance, for parameter values of $\mathcal{A} \sim 3 \times 10^{-6} \mathrm{~s}^{-1}$ and $D \sim 30 \mathrm{~cm}^{2} \mathrm{~s}^{-1}$ in the tachocline, $D_{\mathrm{T}}^{x x} / D_{\mathrm{T}}^{z z} \propto 10^{-4}$ for $k_{y} \sim 10^{-7} \mathrm{~cm}^{-1} \sim 10^{3} / H_{0}$ while $D_{\mathrm{T}}^{x x} / D_{\mathrm{T}}^{z z} \propto 10^{-7}$ for $k_{y} \sim 10^{-9} \mathrm{~cm}^{-1} \sim 10 / H_{0}$. It is important to note that the anisotropy in particle diffusivity due to radial shear, measured by $D_{\mathrm{T}}^{x x} / D_{\mathrm{T}}^{z z} \propto\left(\mathcal{A} / D k_{y}^{2}\right)^{-2 / 3}$, depends on $\mathcal{A}$ itself, and can be much larger than that caused by Coriolis forces alone (which is only a factor of 2). For instance, at the poles, Coriolis forces alone would lead to $D_{\mathrm{T}}^{x x}$ (along the rotation axis) which is twice larger than $D_{\mathrm{T}}^{z z}$. This effect will, however, be easily overwhelmed by a much stronger reduction caused by radial shear with the result $D_{\mathrm{T}}^{x x} \ll D_{\mathrm{T}}^{z z}$.

Thus, shearing results in the anisotropy in particle transport, as well as in turbulence intensity, with less transport in the radial direction. Physically, this result makes sense since it is only through diffusion that the radial shear has any effect on the particle transport in the horizontal plane. In fact, in the 
limit $D \rightarrow 0, D_{\mathrm{T}}^{z z} \rightarrow \infty$ as density fluctuation accumulates on small-scales in the absence of diffusion while $D_{\mathrm{T}}^{x x}$, suffering directly from shearing, remains finite. For finite $D$, the transport in the horizontal plane is affected by shearing only through the enhanced dissipation, and thus less reduced.

As previously noted, the anisotropy in the particle transport $D_{\mathrm{T}}^{x x} / D_{\mathrm{T}}^{z z} \propto\left(\mathcal{A l} / D k_{y}^{2}\right)^{-2 / 3}$ is, however, different from that in turbulence amplitude $\left\langle v_{x}^{2}\right\rangle /\left\langle v_{z}^{2}\right\rangle \sim\left(\mathcal{A} / D k_{y}^{2}\right)^{-1 / 3}$. Furthermore, in comparison with Eq. (24), Eq. (37) indicates $D_{\mathrm{T}}^{z z} \sim$ $\mathcal{A}^{-1}\left(\mathcal{A} / D k_{y}^{2}\right)^{1 / 3}\left\langle v_{z}^{2}\right\rangle$. Thus, the proportionality between $D_{\mathrm{T}}^{z z}$ and $\left\langle v_{z}^{2}\right\rangle$ is not constant, but depends on $\mathcal{A}$. We recall that we have found a similar result for the transport of particle in the vertical direction with $D_{\mathrm{T}}^{x x} \sim \mathcal{A}^{-1}\left\langle v_{x}^{2}\right\rangle$. To see how the shearing affects the effective correlation time $\tau_{z}$ and length scale of turbulent flow $l_{z}$ in the horizontal plane, we let the characteristic velocity of turbulent flow $v_{z}$ in that plane. Then, by comparing the conventional expectation $D_{\mathrm{T}}^{z z} \sim l_{z} v_{z} \sim \tau_{z} v_{z}^{2}$ with our result $D_{\mathrm{T}}^{z z} \sim \mathcal{A}^{-1}\left(\mathcal{A} / D k_{y}^{2}\right)^{1 / 3} v_{z}^{2}$, we obtain $\tau_{z} \sim \mathcal{A}^{-1}\left(\mathcal{A} / D k_{y}^{2}\right)^{1 / 3}$ and $l_{z} \sim \mathcal{A}^{-1}\left(\mathcal{A} / D k_{y}^{2}\right)^{1 / 3} v_{z}$. These results indicate that horizontal turbulence is also subjected to the enhanced decorrelation by shear flow, but only through dissipation $(D \neq 0)$. This is also true in the case of a purely horizontal forcing with $f_{x}=0$, similarly to $\left\langle v_{z}^{2}\right\rangle$ (see the discussion before Eq. (25) in Sect. 3.1). Therefore, while in the presence of non-vanishing dissipation $(D \neq 0)$, the shear flow shortens the effective length scale $l_{z}$ and decorrelation time $\tau_{z}$ in the horizontal plane, the decorrelation time $\tau_{z}$ for horizontal turbulence is still larger than $\tau_{x}$ for vertical turbulence for which the radial shear operates more efficiently.

\subsection{Estimates and implications for light element burning}

When turbulence is driven by plumes, $D_{\mathrm{T}}^{x x} \neq 0$. In this case, Eqs. (27), (29), (33), and (37) suggest that the transport by $\boldsymbol{U}_{m}$ dominates over the transport by turbulence $\left(D_{\text {eff }}>D_{\mathrm{T}}^{x x}\right)$ when

$$
\begin{aligned}
V_{m} L & >\sqrt{D_{\mathrm{T}}^{x x} D_{\mathrm{T}}^{z z}} \sim \frac{\alpha v_{f}^{2}}{\tau_{f} \mathcal{A}^{2}}\left(\frac{\mathcal{A}}{D k_{y}^{2}}\right)^{1 / 3} \\
& \sim\left(\frac{\mathcal{A}}{D k_{y}^{2}}\right)^{1 / 3} D_{\mathrm{T}}^{x x} \sim \frac{\left\langle v_{z}^{2}\right\rangle}{\mathcal{A}} .
\end{aligned}
$$

Here, $L=1 / p$ is the characteristic scale of $\boldsymbol{U}_{m} ; D_{\mathrm{T}}^{x x} / D_{\mathrm{T}}^{z z} \propto$ $\left(\mathcal{A} / D k_{y}^{2}\right)^{-2 / 3}$ and $\left\langle v_{x}^{2}\right\rangle /\left\langle v_{z}^{2}\right\rangle \sim\left(\mathcal{A} / D k_{y}^{2}\right)^{-1 / 3}$ were used. Interestingly, Eq. (38) is easily satisfied as $\mathcal{A}$ (radial shear) increases. This is basically because shearing reduces turbulent particle transport, making it less important compared to the transport by meridional circulation. Higher values of $\mathcal{A}$ (i.e., radial differential rotation) might be more easily realized inside massive stars or in the early evolution of the Sun owing to fast rotation although there has been no observational evidence for a radial shear layer in other stars, similar to the solar tachocline. Furthermore, meridional circulation (EddingtonSweet circulation) itself becomes more efficient in fast rotating stars. Therefore, the transport by meridional circulation could play a crucial role in these stars.
To assess the importance of the transport by a meridional circulation in the Sun, we take $\mathcal{A} \sim 3 \times 10^{-6} \mathrm{~s}^{-1}, k_{y} \sim 10 / H_{0} \sim$ $10^{-9} \mathrm{~cm}^{-1}, D \sim 30 \mathrm{~cm}^{2} \mathrm{~s}^{-1}, \tau_{f} \mathcal{A} \sim 10^{-2}$, and $L \sim H_{0} \sim$ $6 \times 10^{9} \mathrm{~cm}$; thus $\mathcal{A} / D k_{y}^{2} \sim 10^{11}$ and $D_{\mathrm{T}}^{z z} \sim 10^{7} D_{\mathrm{T}}^{x x}$ and $\left\langle v_{z}^{2}\right\rangle \sim 10^{4}\left\langle v_{x}^{2}\right\rangle$. Here, $H_{0} \mathrm{~cm}$ is pressure scale height. We note that in order to be consistent with the depletion of lithium, the total (vertical) diffusivity of chemical elements near the tachocline must be $\sim 20 D \sim 10^{3} \mathrm{~cm}^{2} \mathrm{~s}^{-1}$ (Barnes et al. 1999). Thus, by requiring that the maximum of $D_{\text {eff }}$ and $D_{\mathrm{T}}^{x x}$ be set by this total diffusivity $20 D$, we can consider the following two cases.

1. If lithium is mainly carried by a meridional circulation $\left[D_{\text {eff }} \sim 20 D>D_{\mathrm{T}}^{x x}\right]: D_{\mathrm{T}}^{x x}<20 D$ constrains the amplitude of turbulent velocity to be $v_{x}<10^{-1} \mathrm{~cm} \mathrm{~s}^{-1}$ and $v_{z}<10 \mathrm{~cm} \mathrm{~s}^{-1}$ with $D_{\mathrm{T}}^{z z}<10^{10} \mathrm{~cm}^{2} \mathrm{~s}^{-1}$, Equivalently, the amplitude of forcing should be $v_{f}<10^{-2} / \sqrt{\alpha} \mathrm{cm} \mathrm{s}^{-1}$ (see Eq. (29)). For instance, $v_{f}<10^{-2} \mathrm{~cm} \mathrm{~s}^{-1}$ for $\alpha \sim 1$ and $v_{f}<10^{2} \mathrm{~cm} \mathrm{~s}^{-1}$ for $\alpha \sim 10^{-8}$. Thus, Eq. (33) with $p=1 / L$ implies $V_{m}<10^{6} / L \sim 10^{-4} \mathrm{~cm} \mathrm{~s}^{-1}$. The upper limit on $V_{m}$ is due to the fact that $D_{\text {eff }}$ increases for small $D_{\mathrm{T}}^{x x}$ since $D_{\text {eff }} \propto 1 / D_{\mathrm{T}}^{z z} \propto 1 / D_{\mathrm{T}}^{x x}$. On the other hand, in order for $D_{\text {eff }}>D$ and $D_{T}^{z z} \gg D, V_{m}>D / L \sim 10^{-9} \mathrm{~cm} \mathrm{~s}^{-1}$ and $v_{f} \gg 10^{-6} / \sqrt{\alpha} \mathrm{cm} \mathrm{s}^{-1}$.

2. If lithium is mixed largely by turbulence $\left[D_{\text {eff }}<D_{\mathrm{T}}^{x x} \sim\right.$ 20D]: $D_{\mathrm{T}}^{x x} \sim 20 D$ gives $v_{x} \sim 10^{-1} \mathrm{~cm} \mathrm{~s}^{-1}, v_{z} \sim 10 \mathrm{~cm} \mathrm{~s}^{-1}$, $D_{\mathrm{T}}^{z z} \sim 10^{10} \mathrm{~cm}^{2} \mathrm{~s}^{-1}$, and $v_{f} \sim 10^{-2} / \sqrt{\alpha} \mathrm{cm} \mathrm{s}^{-1}$ (see Eq. (29)). For example, $v_{f} \sim 10^{-2} \mathrm{~cm} \mathrm{~s}^{-1}$ for $\alpha \sim 1$ while $v_{f} \sim 10^{2} \mathrm{~cm} \mathrm{~s}^{-1}$ for $\alpha \sim 10^{-8}$. Thus, Eq. (33) with $p=1 / L$ implies $V_{m}<10^{6} / L \sim 10^{-4} \mathrm{~cm} \mathrm{~s}^{-1}$.

Therefore, in both cases, the upper limit on $V_{m}$ is of order $V_{m} \sim 10^{-4} \mathrm{~cm} \mathrm{~s}^{-1}$ for the chosen parameter values while the typical velocity of the forcing (i.e. plumes) determines whether the transport is mainly via a meridional circulation or via turbulence. However, given the uncertainties in the the amount (filling factor) and strength of plumes penetrating into the tachocline regime (e.g., Rempel 2004), it is not entirely clear as to which of the two plays a more important role. Furthermore, as noted previously, not all the plumes penetrate through the entire tachocline to carry lithium near $R \sim 0.65 R_{\odot}$, which is little below the bottom of the tachocline, for their destruction. The filling factor $\alpha$ for those plumes may be very small. Note that the estimates given above are based on the strong observational constraint on the total vertical diffusivity of lithium (20 times molecular value) in the case when turbulence in the tachocline is driven by plumes only. If there is an additional forcing driving turbulence in the horizontal plane only, with no vertical turbulent flow, the horizontal turbulent diffusivity $D_{\mathrm{H}}$ due to this forcing should be added to the total horizontal turbulent diffusivity as $D_{\mathrm{H}}+D_{\mathrm{T}}^{z z}$. Thus, there would be no restriction on the amplitude of total horizontal turbulent diffusivity (and velocity), and the anisotropy would be enhanced as $D_{\mathrm{T}}^{x x} /\left(D_{\mathrm{T}}^{z z}+D_{\mathrm{H}}\right)$ as compared to $D_{\mathrm{T}}^{x x} / D_{\mathrm{T}}^{z z}$ discussed above. Furthermore, if there are mechanisms inhibiting the transport of lithiums (e.g. magnetic fields, density stratification, etc.), vertical turbulent velocity can take much larger value than the estimated one without causing inconsistency with lithium depletion. 


\section{Discussions and conclusions}

We have presented a self-consistent theory of turbulent transport in the tachocline by taking into account the effect of shearing by the radial differential rotation. Under the physically plausible assumption that turbulence in the tachocline is driven by plumes penetrating from the convection zone, or the instabilities of latitudinal differential rotation, we have demonstrated that shearing can cause significant reduction in the amplitude of turbulent flow and transport of particle and momentum. Specifically, the scalings of these quantities with the shearing rate $\mathcal{A}$ (the radial differential rotation) are as follows: $\left\langle v_{x}^{2}\right\rangle \propto \mathcal{A}^{-1},\left\langle v_{z}^{2}\right\rangle \propto \mathcal{A}^{-1}\left(\mathcal{A} / D k_{y}^{2}\right)^{1 / 3}, v_{\mathrm{T}}^{x x} \propto \mathcal{A}^{-2}, D_{\mathrm{T}}^{x x} \propto \mathcal{A}^{-2}$, and $D_{\mathrm{T}}^{z z} \propto \mathcal{A}^{-2}\left(\mathcal{A} / D k_{y}^{2}\right)^{2 / 3}$. Note that $D k_{y}^{2} / \mathcal{A} \ll 1$ is a small parameter used to derive these results. The overall amplitude of $D_{\mathrm{T}}^{x x}, v_{\mathrm{T}}^{x x}$ and $\left\langle v_{x}^{2}\right\rangle$ vanishes if the forcing is due to the instability of the latitudinal differential rotation with no vertical component of the forcing $\left(f_{x}=0\right)$, which is incompressible $(\nabla \cdot f=0)$. Therefore, for typical parameter values $\mathcal{A} \sim$ $3 \times 10^{-6} \mathrm{~s}^{-1}$ and $D \sim 30 \mathrm{~cm}^{2} \mathrm{~s}^{-1}, \mathcal{A} / D k_{y}^{2} \sim 10^{6}, D_{\mathrm{T}}^{z z} \sim 10^{4} D_{\mathrm{T}}^{x x}$ and $\left\langle v_{z}^{2}\right\rangle \sim 10^{2}\left\langle v_{x}^{2}\right\rangle$ for $k_{y} \sim 10^{-7} \mathrm{~cm}^{-1}$ while $\mathcal{A} / D k_{y}^{2} \sim 10^{11}$, $D_{\mathrm{T}}^{z z} \sim 10^{7} D_{\mathrm{T}}^{x x}$ and $\left\langle v_{z}^{2}\right\rangle \sim 10^{4}\left\langle v_{x}^{2}\right\rangle$ for $k_{y} \sim 10^{-9} \mathrm{~cm}^{-1}$. These results indicate the following:

1. A careful distinction should be made between the amplitude of turbulent flow and turbulent transport of particles which have different scalings with $\mathcal{A}$.

2. The degree of turbulence reduction differs in the horizontal plane and in the vertical direction, with the resulting anisotropy in both turbulence amplitude and particle transport.

3. Anisotropy in the particle transport is stronger with the scaling $D_{\mathrm{T}}^{x x} / D_{\mathrm{T}}^{z z} \propto\left(\mathcal{A} / D k_{y}^{2}\right)^{-2 / 3}$ than that in turbulence amplitude $\left\langle v_{x}^{2}\right\rangle /\left\langle v_{z}^{2}\right\rangle \sim\left(\mathcal{A} / D k_{y}^{2}\right)^{-1 / 3}$.

4. The turbulence quenching and anisotropy due to radial shear can be significantly larger than those caused by Coriolis force.

5. Anisotropic turbulence can be caused by radial differential rotation alone in the tachocline even in the absence of density stratification and magnetic fields.

6. The amplitude of $D_{\mathrm{T}}^{x x}$ and $v_{\mathrm{T}}^{x x}$ is of similar magnitude, implying no significant disparity between particle and momentum transport in the hydrodynamic turbulence considered in the paper.

We have also investigated how the transport by meridional circulation is affected by shearing, by self-consistently computing the horizontal turbulent diffusivity, and then assessed its efficiency compared to turbulent transport. The results indicate that the contribution from a meridional circulation to particle transport increases for a larger shearing rate (i.e. radial differential rotation). Thus, if fast rotating stars contain significant radial shear in the interior, the particle transport by meridional circulation could be crucial to mixing of lithium, and thus to explaining Lithium dip in F stars (Zahn 1992; Schatzman 1993; Pinsonneault 1997). Whether a meridional flow can significantly contribute to mixing at the present Sun or not is not totally clear at the present time, given the uncertainties in the amplitude of meridional circulation, and the scales, amount (filling factor), strength, and depth of the penetration of plumes. Further study on generation and characteristics of plumes penetrating into the tachocline, in particular, a statistical study on the probability distribution function of penetration depth and strength of plumes, will shed some light on this issue.

The results have very interesting implications for the mixing and diffusion in the tachocline. Since the radial differential rotation is largest near the poles and equator, the quenching of the turbulent transport (such as diffusion of chemical elements) will be most prominent near the poles and equator with the latitudinal dependent diffusion and mixing. They may also have an important implication for the long-term dynamics of the solar tachocline. According to our results, a strong radial shear can cause anisotropic turbulent transport of particles in that regime. If similar anisotropy also develops in momentum transport (i.e. eddy viscosity), it can in turn prevent the radiative spreading of the region (Spiegel \& Zahn 1992). That is, the radial shear may have a positive feedback on keeping a strong radial shear in the thin regime. In order to address this problem, the present work should be extended to include the latitudinal differential rotation and to study horizontal turbulent transport of momentum. Of course, there are other alternative mechanisms for maintaining thin tachocline. For instance, it may be through Lorentz force in the case of magnetized tachocline (Rüdiger \& Kitchatinov 1996; Gough \& McIntyre 1998; MacGregor \& Charbonneau 1999). Nevertheless, the aforementioned positive feedback due to shearing can still play an important role in the overall dynamics of the tachocline and thus should be investigated. In the case where the tachocline evolves on a short time scale of order of $1 \mathrm{yr}$ (i.e. fast tachocline), for instance, due to the instability of latitudinal differential rotation, (e.g. Gilman \& Fox 1997; Dikpati \& Gilman 1999; Gilman \& Dikpati 2000; Cally et al. 2003), the overall angular momentum transport in the tachocline should be closely linked to that in the convection zone (Gilman 2000), and other physical effects not included in the paper will play an important role in determining the thickness of the tachocline.

Finally, we note that the analysis in the present paper was limited to purely hydrodynamical turbulence without density stratification and without Coriolis forces in the tachocline in order to clearly identify the effect of radial differential shear. While turbulence quenching and anisotropy in the tachocline can be caused by shearing effect alone, it is important to include the effects of Coriolis forces, density stratification and strong toroidal magnetic fields. Even if the reduction in the transport and anisotropy caused by Coriolis forces alone is likely to be much weaker as compared to those by the radial shear discussed in this paper, it is important to study how Coriolis forces together with radial shear affect the overall dynamics of turbulence and transport in the tachocline. In particular, Coriolis forces may lead to the $\Lambda$ effect, with an interesting consequence in the angular momentum transport in the tachocline. On the other hand, both density stratification and magnetic fields would enhance the anisotropy in turbulence due to radial shear found in the paper, by further reducing transport in the radial direction. In fact, it is the density stratification which is commonly thought to be responsible for anisotropic turbulence in the tachocline. In addition to causing anisotropy, 
magnetic fields may also have different effects on the transport of particles and momentum, which can have a significant implication for explaining apparent, more efficient angular momentum transport than the mixing of lithium over the evolution of the Sun. Note that within the hydrodynamical model considered in the paper, the transport of particle and momentum appears to be comparable. Note also that there are alternative mechanisms to explain these problems including gravity waves (e.g., García Lopez \& Spruit 1991). Furthermore, in the magnetized tachocline, the problem of transport of magnetic flux itself would become of considerable interest for solar dynamos. The work addressing these issues is in progress and will be published in the subsequent papers.

Acknowledgements. The author thanks M. J. Thompson and anonymous referee for useful comments.

\section{References}

Barnes, G., Charbonneau, P., \& MacGregor, K. B. 1999, ApJ, 511, 466 Basri, G. 1985, in Cool stars, stellar systems, and the Sun, ed. M. Zeilik, \& P. M. Gibson, Lect. Notes Phys., 254, 184 (SpringerVerlag)

Biglari, H., Diamond, P. H., \& Terry, P. W. 1990, Phys. Fluids B, 2, 1

Brummell, N. H., Clune, T. L., \& Toomre, J. 2002, ApJ, 570, 825

Burrell, K. H. 1997, Phys. Plasmas, 4, 1499

Busse, F. H. 1983, Geophys. Astrophys. Fluid Dyn., 23, 153

Cally, P. S., Dikpati, M., \& Gilman, P. A. 2003, ApJ, 582, 1190

Chaboyer, B., \& Zhan, J.-P. 1992, A\&A, 253, 173

Charbonneau, P., Christensen-Dalsgaard, J., Henning, R., et al. 1999, ApJ, 527, 445

Dikpati, M., \& Gilman, P. A. 1999, ApJ, 512, 417

Eddington, A. S. 1925, Observatory, 48, 78

García Lopez, R. J., \& Spruit, H. C. 1991, A\&A, 377, 268

Gilman, P. A. 2000, Sol. Phys., 192, 27

Gilman, P. A., \& Fox, P. A. 1997, ApJ, 484, 439

Gilman, P. A., \& Dikpati, M. 2000, ApJ, 528, 552
Gilman, P. A., \& Miesch, M. 2004, ApJ, 611, 568

Gough, D. O., \& McIntyre, M. E. 1998, Nature, 394, 755

Haber, D. A., Hindman, B. W., Toomre, J., et al. 2000, Sol. Phys., 192, 335

Hahm, T. S. 2002, Plasma Phys. Control. Fusion A, 44, 87

Kichatinov, L. L. 1987, Geophys. Astrophys. Fluid Dynamics, 38, 273

Kichatinov, L. L., \& Rüdiger, G. 1993, A\&A, 276, 96

Kichatinov, L. L., Pipin, V. V., \& Rüdiger, G. 1994, Astron. Nachr., 315,157

Kichatinov, L. L., \& Rüdiger, G. 1995, A\&A, 299, 446

Kim, E. 2004, Mod. Phys. Lett. B, 18, 1

Kim, E., \& Dubrulle, B. 2001, Phys. Plasmas, 8, 813

Kim, E., \& Diamond, P. H. 2003, Phys. Rev. Lett., 90, 185006

Kim, E., Diamond, P. H., \& Hahm, T. S. 2004, Phys. Plasmas, 11, 4554

Kim, E., \& Diamond, P. H. 2004, Phys. Plasmas, 11, L77

Kim, E., \& MacGregor, K. B. 2001, ApJ, 556, L117

MacGregor, K. B., \& Charbonneauu, P. 1999, ApJ, 519, 911

McIntyre, M. E. 1989, J. Atmosph. Terrest. Phys., 51, 29

Moffatt, H. K. 1978, Magnetic field generation in electrically conducting fluids (Cambridge: Cambridge University Press)

Pinsonneault, M. 1997, ARA\&A, 35, 357

Pinsonneault, M., Kawaler, S. D., Sofia, S., \& Demarque, P. 1989, ApJ, 338, 424

Rempel, M. 2004, ApJ, 607, 1046

Rogers, T. M., \& Glatzmaier, G. A. 2005, ApJ, 620, 432

Rüdiger, G. 1983, Geophys. Astrophys. Fluid Dynamics, 25, 213

Rüdiger, G. 1989, Differential rotation and stellar convection: Sun and solar-type stars (New York: Gordon and Breach)

Rüdiger, G., \& Kitchatinov, L. L. 1996, ApJ, 466, 1078

Schatzman, F. D. 1993, The stars (Berlin, London: Springer-Verlag)

Spiegel, E. A., \& Zahn, J.-P. 1992, A\&A, 265, 106

Stix, M. 1989, The sun: an introduction (Berlin, London: SpringerVerlag)

Sweet, P. A. 1950, MNRAS, 110, 548

Vogt, H. 1925, Astron. Nachr., 223, 229

Zahn, J.-P. 1992, A\&A, 265, 115 
Eun-jin Kim: Self-consistent theory of turbulent transport in the solar tachocline, Online Material p 1

\section{Online Material}




\section{Appendix A:}

In this Appendix, we show how to derive Eqs. (11)-(14) from Eqs. (6)-(8). By using Eqs. (9) and (10) in (6)-(8), we obtain the following set of equations:

$$
\begin{aligned}
\partial_{t} \hat{v}_{x} & =-i k_{x} \hat{p}+\hat{f}_{x}, \\
\partial_{t} \hat{v}_{y}-\mathcal{A} \hat{v}_{x} & =-i k_{y} \hat{p}+\hat{f}_{y}, \\
\partial_{t} \hat{v}_{z} & =-i k_{z} \hat{p}+\hat{f}_{z}, \\
0 & =k_{x} \hat{v}_{x}+k_{y} \hat{v}_{y}+k_{z} \hat{v}_{z}, \\
\partial_{t} \hat{n} & =-\hat{v}_{x} \partial_{x} n_{0} .
\end{aligned}
$$

Here, $\hat{w}$ for $w=v_{i}, n, p$ and $f$ is defined as

$\hat{w} \equiv \tilde{w} \exp \left[D\left(k_{\mathrm{H}}^{2} t+k_{x}^{3} / 3 k_{y} \mathcal{A}\right)\right]$

where $k_{\mathrm{H}}^{2}=k_{y}^{2}+k_{z}^{2}$. First, Eq. (A.5) can readily be integrated to give Eq. (14). Now, to solve coupled Eqs. (A.1)-(A.4), we introduce a new time variable $\tau=k_{x} / k_{y}+\mathcal{A} t$ and rewrite them as:

$$
\begin{aligned}
\mathcal{A} \partial_{\tau} \hat{v}_{x} & =-i \tau k_{y} \hat{p}+\hat{f}_{x}, \\
\mathcal{A} \partial_{\tau} \hat{v}_{y}-\mathcal{A} \hat{v}_{x} & =-i k_{y} \hat{p}+\hat{f}_{y}, \\
\mathcal{A} \partial_{\tau} \hat{v}_{z} & =-i k_{z} \hat{p}+\hat{f}_{z}, \\
0 & =\tau \hat{v}_{x}+k_{y} \hat{v}_{y}+\frac{k_{z}}{k_{y}} \hat{v}_{z} .
\end{aligned}
$$

A straightforward, but rather long, algebra then gives us the solutions in the following form:

$$
\begin{aligned}
\hat{v}_{x}(\tau)= & \frac{1}{\gamma+\tau^{2}} \int^{\tau} \mathrm{d} \tau_{1} h_{1}\left(\tau_{1}\right) \\
\hat{v}_{z}(\tau)= & -\frac{\beta \tau}{\gamma} \hat{v}_{x}+\int^{\tau} \mathrm{d} \tau_{1} \frac{1}{\gamma}\left[h_{2}\left(\tau_{1}\right)-\frac{\beta}{\gamma^{1 / 2}}\left(\tan ^{-1} \frac{\tau}{\sqrt{\gamma}}\right.\right. \\
& \left.\left.-\tan ^{-1} \frac{\tau_{1}}{\sqrt{\gamma}}\right) h_{1}\left(\tau_{1}\right)\right], \\
\hat{v}_{y}(\tau)= & -\tau \hat{v}_{x}(\tau)-\beta \hat{v}_{z}(\tau),
\end{aligned}
$$

where $\beta=k_{z} / k_{y}, \gamma=1+\beta^{2}, h_{1}=\left(1+\beta^{2}\right) \hat{f}_{x}-\tau_{1} \hat{f}_{y}-\tau_{1} \beta \hat{f}_{z}$, and $h_{2}=-\beta \hat{f}_{y}+\hat{f}_{z}$. Finally, going back to the original variable $k_{x}=k_{y} \tau$, we obtain Eqs. (11)-(13).

\section{Appendix B:}

In this Appendix B, we provide a few key steps leading to Eqs. (26) and (27). From Eqs. (9)-(13), (15)-(17), and (21), we obtain

$$
\begin{aligned}
\left\langle v_{x} v_{y}\right\rangle= & \frac{\tau_{f}}{(2 \pi)^{3}} \\
& \times \int \mathrm{d}^{3} k \mathrm{~d} t_{1} \mathrm{~d}^{3} k_{1} \mathrm{e}^{-2 D Q\left(t, t_{1}\right)} \hat{g}\left(\boldsymbol{k}, t ; \boldsymbol{k}_{1}, t_{1}\right)\left\{-\phi_{12}\left(\boldsymbol{k}_{1}\right) \frac{k_{y}^{3} k_{z}}{k^{2} k_{\mathrm{H}}^{2}}\right. \\
& +\phi_{11}\left(\boldsymbol{k}_{1}\right) \frac{k_{y}^{2}}{k^{2}}\left[-\frac{k_{x} k_{y}}{k^{2}}+\frac{k_{z}^{2} k_{x} k_{y}}{k^{2} k_{\mathrm{H}}^{2}}\right. \\
& \left.\left.+\frac{k_{z}^{2} k_{y}}{\left|k_{\mathrm{H}}\right|^{3}}\left(\tan ^{-1}\left(\frac{k_{x}}{\left|k_{\mathrm{H}}\right|}\right)-\tan ^{-1}\left(\frac{k_{1 x}}{\left|k_{\mathrm{H}}\right|}\right)\right)\right]\right\}
\end{aligned}
$$

To evaluate the integral over time $t_{1}$ in Eq. (B.1), we introduce a new time variable $\tau=k_{x} / k_{y}=k_{1 x} / k_{1 y}+\mathcal{A}\left(t-t_{1}\right)$ and express
$D Q\left(t, t_{1}\right)=\xi\left[\tau^{3} / 3+\gamma \tau-\left(a^{3} / 3+\gamma a\right)\right]$, where $\gamma=k_{\mathrm{H}}^{2} / k_{y}^{2}, a=$ $k_{1 x} / k_{1 y}$, and $\xi=D k_{y}^{2} / \mathcal{A}$. Then, by taking the limit $\xi \rightarrow 0$ in the $\tau$ integral, by using $\left\langle v_{x} v_{y}\right\rangle=-v_{\mathrm{T}}^{x x} \partial_{x} U_{0}=v_{\mathrm{T}}^{x x} \mathcal{A}$, and by assuming $\psi_{i j}\left(k_{y}\right)=\psi_{i j}\left(-k_{y}\right)$, we obtain Eq. (26) to leading order in $\xi \ll 1$.

To obtain Eq. (27), we substitute Eq. (11) in (14) and change the order of time integrals to obtain

$$
\begin{aligned}
n^{\prime}(\boldsymbol{k}, t)= & -\frac{\left(\partial_{x} n_{0}\right)}{\mathcal{A}} \int \mathrm{d}^{3} k \mathrm{~d} t_{1} \mathrm{~d}^{3} k_{1} \mathrm{e}^{-D Q\left(t, t_{1}\right)} \hat{g}\left(\boldsymbol{k}, t ; \boldsymbol{k}_{1}, t_{1}\right) \\
& \times \frac{k_{y}}{\left|k_{\mathrm{H}}\right|}\left[\tan ^{-1}\left(\frac{k_{x}}{\left|k_{\mathrm{H}}\right|}\right)-\tan ^{-1}\left(\frac{k_{1 x}}{\left|k_{\mathrm{H}}\right|}\right)\right] \tilde{h}_{1}\left(\boldsymbol{k}_{1}, t_{1}\right) .
\end{aligned}
$$

Then, from Eqs. (9)-(11), (15)-(17), and (B.2), we obtain

$$
\begin{aligned}
\left\langle n^{\prime} v_{x}\right\rangle= & -\frac{\tau_{f}\left(\partial_{x} n_{0}\right)}{(2 \pi)^{3}} \int \mathrm{d}^{3} k \mathrm{~d} t_{1} \mathrm{~d}^{3} k_{1} \mathrm{e}^{-2 D Q\left(t, t_{1}\right)} \hat{g}\left(\boldsymbol{k}, t ; \boldsymbol{k}_{1}, t_{1}\right) \phi_{11}\left(\boldsymbol{k}_{1}\right) \\
& \times \frac{k_{y}^{3}}{k^{2}\left|k_{\mathrm{H}}\right|}\left[\tan ^{-1}\left(\frac{k_{x}}{\left|k_{\mathrm{H}}\right|}\right)-\tan ^{-1}\left(\frac{k_{1 x}}{\left|k_{\mathrm{H}}\right|}\right)\right],
\end{aligned}
$$

to leading order in $\xi \ll 1$. Again, $t_{1}$-time integral in Eq. (B.3) can easily be performed in terms of a new time variable $\tau=$ $k_{x} / k_{y}=k_{1 x} / k_{1 y}+\mathcal{A}\left(t-t_{1}\right)$, with the result given in Eq. (27). 